\title{
A Phenomenological Extension of the Newtonian Gravity
}

\author{
Heinz Dehnen \\ Fachbereich Physik, Universität Konstanz, Konstanz, Germany \\ Email: heinz.dehnen@uni-konstanz.de
}

How to cite this paper: Dehnen, H. (2019) A Phenomenological Extension of the Newtonian Gravity. International Journal of Astronomy and Astrophysics, 9, 63-70. https://doi.org/10.4236/ijaa.2019.91006

Received: January 25, 2019

Accepted: March 16, 2019

Published: March 19, 2019

Copyright (C) 2019 by author(s) and Scientific Research Publishing Inc. This work is licensed under the Creative Commons Attribution International License (CC BY 4.0).

http://creativecommons.org/licenses/by/4.0/

\begin{abstract}
Following the idea of our previous paper we distinguish also in the case of Newtonian gravity as in the electrodynamics between extensive and intensive field quantities. Between both, a "material" quantity produced e.g. by vacuum polarisations induced by the gravitational field strength itself is mediated. It acts in such a way that it amplifies the field strength in weak gravitational fields and reduces the field strength in strong gravitational fields following Lenz's rule. Newton's gravity is valid only in a very large intermediate range of middle field strength $\boldsymbol{F}\left(\left|\boldsymbol{F}_{0}\right| \ll|\boldsymbol{F}| \ll\left|\boldsymbol{F}_{1}\right|\right), \quad \boldsymbol{F}_{0}$ and $\boldsymbol{F}_{1}$ critical field strengths. In this way dark matter and black holes may be avoidable.
\end{abstract}

\section{Keywords}

Flat Rotation Curves, MOND, Tully-Fisher Law, Avoiding Singularities, Vacuum-Polarisations, Lenz's Rule

\section{Introduction}

In a previous paper [1] we have proposed in view of the dark matter problem a modification of the Newtonian gravity theory in such a way that the assumption of the existence of dark matter is not necessary. In detail we have given a theoretical explanation of MOND [2] following the structure of the electrodynamics assuming also in the case of Newtonian gravity a difference between intensive and extensive field quantities. The intensive field quantity described by $F_{i}$ obeys the homogeneous field equation ${ }^{1}$

$$
F_{i \mid j}-F_{j \mid i}=0 \Rightarrow F_{i}=-\phi_{i},
$$

where $\phi$ is the gravitational potential; simultaneously $F_{i}$ determines the ${ }^{1}$ We use the tensor calculus including the sum convention and restrict ourselves to Cartesian coordinates for simplicity; $\mid i$ means the partial derivative $\frac{\partial}{\partial x_{i}}$. 
gravitational force on a massive point particle (mass $m$ ) at the position $x_{i}(t)$ according to $\left(\cdot=\frac{\mathrm{d}}{\mathrm{d} t}\right)$

$$
\ddot{x}_{i}=F_{i}
$$

using the weak equivalence principle. Thus $F_{i}$ is the field strength. In consequence of (1) and (2) energy conservation is guaranteed for $\phi_{t} \equiv 0$. On the other hand there exists the extensive field quantity $G_{i}$ determined by the mass density $\rho$ of the matter distribution according to the inhomogeneous field equation

$$
G_{i \mid i}=-4 \pi G \rho
$$

( $G$ Newtonian gravitational constant). Accordingly $G_{i}$ is the field excitation, because it is determined by the excitation Equation (3). Between both quantities the "material" equation is mediated:

$$
F_{i}=\gamma G_{i},
$$

with the "material" quantity $\gamma$, which may depend on the field strength itself in consequence e.g. of induced vacuum polarisations. In our previous paper we have assumed

$$
\gamma=\frac{\left|\boldsymbol{F}_{0}\right|}{|\boldsymbol{F}|}+1
$$

where $\left|\boldsymbol{F}_{0}\right|$ is a critical field strength, under which the value of $\gamma$ increases drastically, so that the field strength $|\boldsymbol{F}|$ increases compared with the Newtonian case $(\gamma=1)$, and the assumption of dark matter will be superfluous.

In the present paper we show that the ansatz (5) can be enlarged in a very simple way in such a direction that the field strength $F_{i}$ can also not be larger than a critical field strength $\left|F_{1}\right|$ also in consequence of vacuum polarisations so that singularities (e. g. black holes) may be avoided. Doing this we enlarge relation (5) to

$$
\gamma=\frac{\left|\boldsymbol{F}_{0}\right|}{|\boldsymbol{F}|}+\sqrt{1-\left(\frac{|\boldsymbol{F}|}{\left|\boldsymbol{F}_{1}\right|}\right)^{2}} \quad \text { with }\left|\boldsymbol{F}_{1}\right| \gg\left|\boldsymbol{F}_{0}\right| .
$$

This procedure corresponds exactly to the idea of Born and Infeld in the electrodynamics [3] avoiding there electromagnetic field strengths larger than a critical value. Similar impressive influences of vacuum polarisations and fluctuations on the classical physics are e.g. the Casimir effect [4] and the Scharnhorst-Barton effect [5].

According to our proposal (6) we assume that the gravitational field strength will be weakened by increasing field-strength and enforced by decreasing field-strength in consequence e.g. of vacuum polarisations induced by the gravitational field-strength itself following Lenz's rule. Herewith Lenz's fundamental rule is implemented in the whole range of field strengths. ${ }^{2}$ The Newtonian grav${ }^{1}$ Usually Lenz's rule is connected with time-variable electromagnetic fields. But it has a deeper meaning for all physical systems. If induced quantities, induced by time-variations or variable field strengths in space, would reinforce the original cause, the system would be unstable. Thus Lenz's rule is rather a general stability law. 
ity is valid in the very large intermediate range $\left|\boldsymbol{F}_{0}\right| \ll|\boldsymbol{F}| \ll\left|\boldsymbol{F}_{1}\right|$ where $\gamma \simeq 1$ is valid and where also the Newtonian gravitational constant $G$ is determined. ${ }^{3}$ The value of $\left|\boldsymbol{F}_{0}\right|$ amounts to $2 \times 10^{-8} \mathrm{~cm} / \mathrm{sec}^{2}$ in view of the flat rotation curves of the spiral galaxies (see discussions in [1] and footnote 5), whereas the value of $\left|F_{1}\right|$ may be of the order of $10^{30} \mathrm{~cm} / \mathrm{sec}^{2}$, where the vacuum will be unstable e.g. with respect to spontaneous particle-antiparticle generations. ${ }^{4}$ Of course an exact theoretical derivation of the ansatz (6) does not exist until now because a quantum theory of gravity is missing. In a quantum theory of gravity vacuum polarisation effects would be included, so that a relation like (6), comparable with the running coupling constant in the non-Abelian QCD, would be expectable in the classical limit. In the electromagnetic case Heisenberg and Euler could show that the Born-Infeld ansatz follows from the quantum electrodynamics [6]. In the same sense we consider also our proposal as a phenomenological extension of gravity taking into account expectable quantum effects on the classical level.

\section{The Integration Procedure}

For solving the equation of motion (2) for a point-like test particle the knowledge of the field strength $\boldsymbol{F}$ is necessary. For this we find from the field Equation (1) and Equation (3) with the use of (4) for the potential $\phi$ the differential equation:

$$
\phi_{i \mid i}-\frac{\gamma_{\mid i}}{\gamma} \phi_{i}=4 \pi G \rho \gamma
$$

Herein $\gamma$ is given by (see (6))

$$
\gamma=\frac{\left|\boldsymbol{F}_{0}\right|}{\sqrt{\phi_{j} \phi_{j}}}+\sqrt{1-\frac{\phi_{j} \phi_{j}}{\left|\boldsymbol{F}_{1}\right|^{2}}} .
$$

Now we restrict ourselves for simplicity to the centrally symmetric case of a mass-sphere of mass $M$ with constant density $\rho$ and radius $R$ and consider at first the area outside the sphere, i.e. $r>R$. Than the field Equation (7) reads $\left({ }^{\prime}=\frac{\partial}{\partial r}\right)$ :

$$
\phi^{\prime \prime}+\frac{2}{r} \phi^{\prime}=\frac{\gamma^{\prime}}{\gamma} \phi^{\prime}
$$

with the first integral:

$$
\phi^{\prime} r^{2}=A \gamma, \quad A=\text { const. }
$$

( $A$ integration constant). With

$$
\gamma=\frac{\left|\boldsymbol{F}_{0}\right|}{\phi^{\prime}}+\sqrt{1-\frac{\phi^{\prime 2}}{\left|\boldsymbol{F}_{1}\right|^{2}}}
$$

according to (8) we obtain from (10) the following quadratic equation for $\phi^{\prime 2}$ :

${ }^{3}$ General Relativity would be also valid only in this intermediate range.

${ }^{4} \mathrm{By}$ this acceleration an electron reaches approximately a distance of a Compton wave length during a Compton time necessary for spontaneous electron-positron pair creation. 


$$
\left(\phi^{\prime 2} r^{2}-\left|\boldsymbol{F}_{0}\right| A\right)^{2}=A^{2} \phi^{\prime 2}\left(1-\frac{\phi^{\prime 2}}{\left|\boldsymbol{F}_{1}\right|^{2}}\right)
$$

with the solution:

$$
\phi^{\prime 2}=\frac{1}{2} \frac{A^{2}+2\left|\boldsymbol{F}_{0}\right| A r^{2}}{r^{4}+A^{2} /\left|\boldsymbol{F}_{1}\right|^{2}}+\sqrt{\frac{1}{4}\left(\frac{A^{2}+2\left|\boldsymbol{F}_{0}\right| A r^{2}}{r^{4}+A^{2} /\left|\boldsymbol{F}_{1}\right|^{2}}\right)^{2}-\frac{A^{2}\left|\boldsymbol{F}_{0}\right|^{2}}{r^{4}+A^{2} /\left|\boldsymbol{F}_{1}\right|^{2}}} .
$$

For $r \rightarrow \infty$ we obtain

$$
\phi^{\prime}=\sqrt{A\left|\boldsymbol{F}_{0}\right|} \frac{1}{r},
$$

so that the flat rotation curves of the spiral galaxies are guaranteed with the constant orbital velocity $v(r \rightarrow \infty)=\left(A\left|\boldsymbol{F}_{0}\right|\right)^{1 / 4} .^{5}$ This is in accordance with our previous paper and confirms also the Tully-Fisher law [7]. The exact value of the constant $A$ can be only determined by the connection of the solution (13) with the inner one for $r<R$, see Equation (28).

Thus we consider now the case $r<R$. With the abbreviation

$$
C=4 \pi G \rho
$$

we obtain from (7) the differential equation

$$
\phi_{i \mid i}-\frac{\gamma_{\mid i}}{\gamma} \phi_{i}-C \gamma=0
$$

which goes over in the centrally symmetric case into:

$$
\left(\frac{\phi^{\prime}}{\gamma}\right)^{\prime}+\frac{2}{r} \frac{\phi^{\prime}}{\gamma}=C
$$

We solve this inhomogeneous differential equation by the method of the variation of the constants. The solution of the homogeneous equation belonging to (17) is already known and given by (10) as

$$
\phi^{\prime}=\frac{\tilde{A} \gamma}{r^{2}},
$$

where $\tilde{A}$ is now the integration constant, which is to vary for solving (17). This gives the differential equation for $\tilde{A}$

$$
\tilde{A}^{\prime}=C r^{2}
$$

with the solution

$$
\tilde{A}=\frac{1}{3} C r^{3}+B
$$

( $B$ new integration constant), so that Equation (18) goes over into:

$$
\phi^{\prime}=\left(\frac{1}{3} C r^{3}+B\right) \frac{\gamma}{r^{2}},
$$

where $\gamma$ is finally given by (11). Herewith we obtain once more a quadratic equation for $\phi^{\prime 2}$ with the solution:

${ }^{5}$ Herewith the value $\left|\boldsymbol{F}_{0}\right|$ can be estimated, c.f. (28) and [1]. 


$$
\begin{aligned}
\phi^{\prime 2}= & \frac{1}{2} \frac{\left(\frac{1}{3} C r^{3}+B\right)\left(2\left|\boldsymbol{F}_{0}\right| r^{2}+\frac{1}{3} C r^{3}+B\right)}{r^{4}+\left(\frac{1}{3} C r^{3}+B\right)^{2} /\left|\boldsymbol{F}_{1}\right|^{2}} \\
& +\left[\frac{1}{4} \frac{\left(\frac{1}{3} C r^{3}+B\right)^{2}\left(2\left|\boldsymbol{F}_{0}\right| r^{2}+\frac{1}{3} C r^{3}+B\right)^{2}}{\left(r^{4}+\left(\frac{1}{3} C r^{3}+B\right)^{2} /\left|\boldsymbol{F}_{1}\right|^{2}\right)^{2}}-\frac{\left|\boldsymbol{F}_{0}\right|^{2}\left(\frac{1}{3} C r^{3}+B\right)^{2}}{r^{4}+\left(\frac{1}{3} C r^{3}+B\right)^{2} /\left|\boldsymbol{F}_{1}\right|^{2}}\right]^{1 / 2}
\end{aligned}
$$

Setting $C=0$ we obtain the solution (13), where $B$ plays the role of $A$. Accordingly $B$ has the meaning of

$$
B=M_{0} G,
$$

where $M_{0}$ is the mass value of an additional central point mass (see Equation (28)). For $r \rightarrow 0$ one gets for the case $B \neq 0$

$$
\phi^{\prime 2}(r \rightarrow 0)=\frac{1}{2}\left|\boldsymbol{F}_{1}\right|^{2}\left[1+\sqrt{1-4\left|\boldsymbol{F}_{0}\right|^{2} /\left|\boldsymbol{F}_{1}\right|^{2}}\right] .
$$

Obviously this is the solution for an additional point mass at $r=0$, where the field-strength possesses no singularity but reaches its maximum possible value $\left|\boldsymbol{F}_{1}\right|$ for $\left|\boldsymbol{F}_{0}\right| /\left|\boldsymbol{F}_{1}\right| \ll 1$ independently of the mass value $M_{0}$. Therefore also the existence of black holes should be avoidable.

Avoiding in the following this central point mass we have to set $B=0$. Herewith it follows from (22):

$$
\begin{aligned}
\phi^{\prime 2}= & \frac{1}{2} \frac{\frac{1}{3} C r^{3}\left(2\left|\boldsymbol{F}_{0}\right| r^{2}+\frac{1}{3} C r^{3}\right)}{r^{4}+\left(\frac{1}{3} C r^{3}\right)^{2} /\left|\boldsymbol{F}_{1}\right|^{2}} \\
& +\left[\frac{1}{4} \frac{\left(\frac{1}{3} C r^{3}\right)^{2}\left(2\left|\boldsymbol{F}_{0}\right| r^{2}+\frac{1}{3} C r^{3}\right)^{2}}{\left(r^{4}+\left(\frac{1}{3} C r^{3}\right)^{2} /\left|\boldsymbol{F}_{1}\right|^{2}\right)^{2}}-\frac{\left(\frac{1}{3} C r^{3}\left|\boldsymbol{F}_{0}\right|\right)^{2}}{r^{4}+\left(\frac{1}{3} C r^{3}\right)^{2} /\left|\boldsymbol{F}_{1}\right|^{2}}\right]^{1 / 2}
\end{aligned}
$$

Now we obtain for $r \rightarrow 0$ after inserting of $C$ according to (15) $\left(M=\frac{4 \pi}{3} \rho R^{3}\right)$

$$
\phi^{\prime}(r \rightarrow 0)=\left(\frac{M G}{R^{3}}\left|\boldsymbol{F}_{0}\right|\right)^{1 / 2} \sqrt{r},
$$

which means that the orbital velocity $v$ has the value

$$
v(r \rightarrow 0)=\left(M G\left|\boldsymbol{F}_{0}\right|\right)^{1 / 4}\left(\frac{r}{R}\right)^{3 / 4} .
$$

This is greater than the Newtonian one by the factor $\left(\frac{\left|\boldsymbol{F}_{0}\right| R^{3}}{M G r}\right)^{1 / 4}$. Therefore 
the high star velocities near the center of the galaxy should be discussed newly avoiding the assumption of a black hole in the galaxy center.

Finally we have to determine the meaning of the integration constant $A$ in the solution (13). It follows from the condition, that the solution (25) for $r<R$ goes steadily over into the solution (13) for $r>R$ at $r=R$. On this way one finds immediately

$$
A=M G .
$$

Now we can give the complete solution for the massive sphere of radius $R$ and mass $M$. It is valid for $r \leq R$ (see (25)):

$$
\begin{aligned}
\phi^{\prime 2}= & \frac{1}{2} \frac{M G \frac{r}{R^{3}}\left(M G \frac{r}{R^{3}}+2\left|\boldsymbol{F}_{0}\right|\right)}{1+\left(M G \frac{r}{R^{3}} /\left|\boldsymbol{F}_{1}\right|\right)^{2}} \\
& +\left[\frac{1}{4} \frac{\left(M G \frac{r}{R^{3}}\right)^{2}\left(M G \frac{r}{R^{3}}+2\left|\boldsymbol{F}_{0}\right|\right)^{2}}{\left(1+\left(M G \frac{r}{R^{3}} /\left|\boldsymbol{F}_{1}\right|\right)^{2}\right)^{2}}-\frac{\left(M G \frac{r}{R^{3}}\left|\boldsymbol{F}_{0}\right|\right)^{2}}{1+\left(M G \frac{r}{R^{3}} /\left|\boldsymbol{F}_{1}\right|\right)^{2}}\right]^{1 / 2},
\end{aligned}
$$

and for $r \geq R$ (see (13)):

$$
\begin{aligned}
\phi^{\prime 2}= & \frac{1}{2} \frac{\frac{M G}{r^{2}}\left(\frac{M G}{r^{2}}+2\left|\boldsymbol{F}_{0}\right|\right)}{1+\left(\frac{M G}{r^{2}} /\left|\boldsymbol{F}_{1}\right|\right)^{2}} \\
& +\left[\frac{1}{4}\left(\frac{\frac{M G}{r^{2}}\left(\frac{M G}{r^{2}}+2\left|\boldsymbol{F}_{0}\right|\right)}{1+\left(\frac{M G}{r^{2}} /\left|\boldsymbol{F}_{1}\right|\right)^{2}}\right)^{2}-\frac{\left(\frac{M G}{r^{2}}\left|\boldsymbol{F}_{0}\right|\right)^{2}}{1+\left(\frac{M G}{r^{2}} /\left|\boldsymbol{F}_{1}\right|\right)^{2}}\right]^{1 / 2} .
\end{aligned}
$$

The square root of (29) and (30) is given by (32) and (33). In the case of $R \rightarrow 0$ the solution (29) results in the finite value (compare (24))

$$
\phi^{\prime 2}=\left|\boldsymbol{F}_{1}\right|^{2} \quad \text { for }\left|\boldsymbol{F}_{0}\right| /\left|\boldsymbol{F}_{1}\right| \ll 1,
$$

so that black holes may be avoided, although the field strength is very high. But for confirming this consequence finally a general relativistic investigation of the situation may be necessary.

\section{Alternative Solution of the Centrally Symmetric Case}

Considering the Equation (4) and Equation (6) one can solve these with respect to $F_{i}$ in the centrally symmetric case, i.e. with respect to $\phi^{\prime}$. After that one can insert for $\boldsymbol{G}$ the Newtonian expression $\left|\boldsymbol{F}_{N}\right|$ according to (3). In the centrally symmetric case the condition (1) is fulfilled as one can test easily. In this way one finds: 


$$
\begin{aligned}
\phi^{\prime}= & \sqrt{\frac{1}{2}}\left[\left\{\frac{1}{2} \frac{\left|\boldsymbol{F}_{N}\right|\left(\left|\boldsymbol{F}_{N}\right|+2\left|\boldsymbol{F}_{0}\right|\right)}{1+\left(\left|\boldsymbol{F}_{N}\right| /\left|\boldsymbol{F}_{1}\right|\right)^{2}}+\frac{\left|\boldsymbol{F}_{N}\right|\left|\boldsymbol{F}_{0}\right|}{\sqrt{1+\left(\left|\boldsymbol{F}_{N}\right| /\left|\boldsymbol{F}_{1}\right|\right)^{2}}}\right\}^{1 / 2}\right. \\
& \left.+\left\{\frac{1}{2} \frac{\left|\boldsymbol{F}_{N}\right|\left(\left|\boldsymbol{F}_{N}\right|+2\left|\boldsymbol{F}_{0}\right|\right)}{1+\left(\left|\boldsymbol{F}_{N}\right| /\left|\boldsymbol{F}_{1}\right|\right)^{2}}-\frac{\left|\boldsymbol{F}_{N}\right|\left|\boldsymbol{F}_{0}\right|}{\sqrt{1+\left(\left|\boldsymbol{F}_{N}\right| /\left|\boldsymbol{F}_{1}\right|\right)^{2}}}\right\}^{1 / 2}\right] .
\end{aligned}
$$

For the mass-sphere with constant mass density $\rho$ and radius $R$ one has to insert

$$
\text { for } r<R: \quad\left|\boldsymbol{F}_{N}\right|=\frac{M G}{R^{3}} r \text { and for } r>R: \quad\left|\boldsymbol{F}_{N}\right|=\frac{M G}{r^{2}} .
$$

Squaring of (32) results into (29) and (30).

We mention however explicitly that this procedure is in general only applicable in the centrally symmetric case because of the condition (1) and in the case, where the mass density distribution $\rho$ is not determined by the gravitational field strength $F_{i}$ itself. For the latter case, where $\rho$ is determined e.g. by the Euler equation

$$
\rho\left(v_{i \mid t}+v_{i \mid k} v_{k}\right)=\rho F_{i}-p_{\mid i}
$$

( $p(\rho)$ pressure of the substratum) as e.g. in stars and galaxies or polytropic gas spheres the procedure of chapt. 2 is to be used.

\section{Conclusion}

Considering induced vacuum polarisations we could show in the framework of Newtonian gravity, that the assumption of dark matter and the existence of gravitational field singularities can be avoided. However our proposal discussed in this paper should be translated finally into a general relativistic form. This is not possible in an immediate way, because in the general theory of relativity the distinction between extensive and intensive field quantities is impossible. Therefore the modification of the theory has to start from the very beginning by a modification of the Lagrangian in the form of a $f(R)$-theory [8] as it is done also in the Born-Infeld electrodynamics. This will be our further aim.

\section{Conflicts of Interest}

The author declares no conflicts of interest regarding the publication of this paper.

\section{References}

[1] Dehnen, H. (2018) Newtonian Gravity Reformulated. IJTP, 57, 1404.

[2] Milgrom, M. (1994) Dynamics with a Nonstandard Inertia-Acceleration Relation: An Alternative to Dark Matter in Galactic Systems. Annals of Physics, 229, 384-415. https://doi.org/10.1006/aphy.1994.1012

Bekenstein, J.D. (2006) The Modified Newtonian Dynamics-MOND and Its Implications for New Physics. Contemporary Physics, 47, 387-483. 
https://doi.org/10.1080/00107510701244055

[3] Born, M. and Infeld, L. (1934) Foundations of the New Field Theory. Proceedings of the Royal Society A, 144, 425. https://doi.org/10.1098/rspa.1934.0059

[4] Casimir, H. (1948) On the Attraction between two Perfectly Conducting Plates. Proceedings van de Koninklijke Nederlandse Akademie van Wetenschappen, B51, 793.

[5] Scharnhorst, K. and Barton, G. (1993) QED between Parallel Mirrors: Light Signals Faster Than c, or Amplified by the Vacuum. Journal of Physics A, 26, 2037. https://doi.org/10.1088/0305-4470/26/8/024

[6] Heisenberg, W. and Euler, H. (1936) Folgerungen aus der Diracschen Theorie des Positrons. Zeitschrift für Physik, 98, 714-732. https://doi.org/10.1007/BF01343663

[7] Tully, R.B. and Fisher, J.R. (1977) A new Method of Determining Distances to Galaxies Astronomy \& Astrophysics, 54, 661.

[8] Buchdahl, H.A. (1970) Non-Linear Lagrangians and Cosmological Theory. Monthly Notices of the Royal Astronomical Society, 150, 1-8.

https://doi.org/10.1093/mnras/150.1.1 